\title{
Medical controversies and systematic reviews: the heat and the light
}

$\mathrm{T}$

There are many ways to prove that doing a systematic review in order to acquire the best evidence to apply to medical practice is better than to continuing to practicing the old-fashioned way of preparing overviews, with no methods.

A systematic review implies in the use of reproducible methods. It also implies the intention to prevent bias in the process of including and excluding the clinical trials for the statistical summary (meta-analysis), to establish criteria to include well-designed and wellconducted trials, and the intention to beat the publication bias phenomenon.

But imagine that someone, for instance, is interested in the effect of the streptokinase on the mortality rate as a consequence of the acute myocardial infarction. This was the case for Lau et al, 1992 (1). After a careful search for relevant trials in the literature, and submitting the trials that were found to the application of inclusion and exclusion criteria described in the publication, 35 trials were selected. Of these, five described significant reduction in the mortality due infarctions, but 30 did not. However, the typical odds ratio had shown a significant mortality rate reduction that was already detectable in the

* $M D, P h D, M C E$

Chairman, Department of Internal Medicine, Escola

Paulista de Medicina, Editor, São Paulo Medical Journal. cumulative odds ratio in the early 1970 s, about 20 years before, this presenting Lau's systematic review.

Now suppose that a group of medical student, are sent to a good library to research the same question, had enough skills to find the same 35 trials select using the criteria established in the systematic review. Each one may come out depending on their particular skills and determinations with $1,2,3,4,5,6$, up to 35 trials, or the combination of 35 trials 2 by 2,35 trails 3 by 3,35 trials 4 by 4 and so on. At the end of the day they would be faced $34,700,000$ different sets of clinical trials, and of course, a great probability of different opinions. That is usually called medical controversy.

Thus it is my understanding that doing systematic review is a good way of preclude the heat of medical controversies, and to shed more light on improving the practice of medicine.

\section{REFERENCES}

1. Lau J, Antman EM, Jimenez-Silva J, Kupelnick B, Mosteller F, Chalmers TC. Cumulative meta-analysis of therapeutic trials for myocardial infarction. N Engl J Med 1992; 327:24854. 